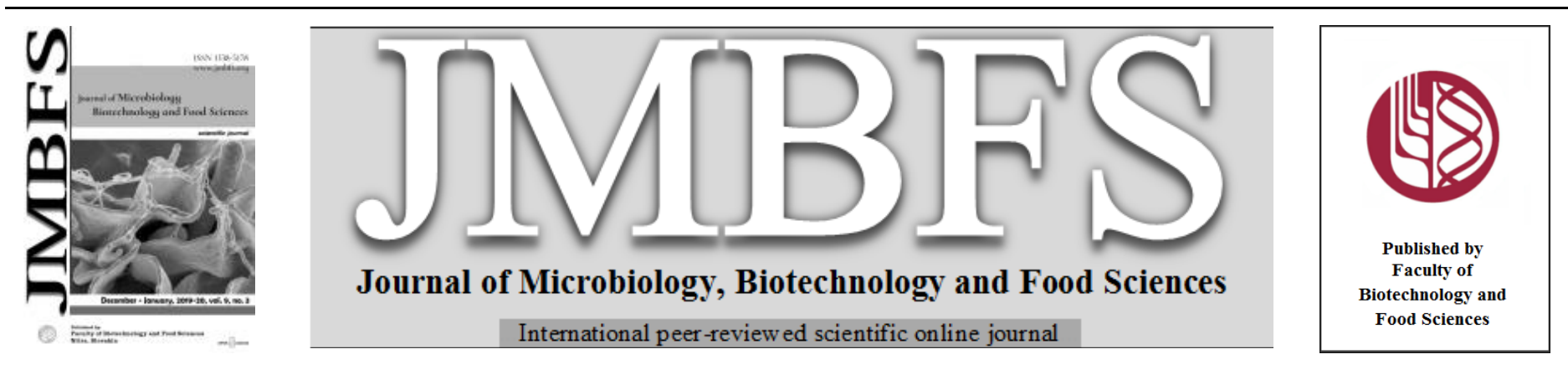

\title{
MULTIPLE ANTIBIOTIC RESISTANCE ACTIVATOR (MarA) OF THE FAMILY ENTEROBACTERIACEAE: STRUCTURE AND CONSERVATION IN Salmonella enterica SUBSP. enterica SEROVAR TYPHIMURIUM
}

\author{
Muhammad Tausif Chaudhry*, Raheela Chaudhry \\ Address(es): \\ CMD, National Physical and Standards Laboratory, PCSIR, Islamabad, Pakistan.
}

*Corresponding author: tausif chaudhry@yahoo.com

doi: 10.15414/jmbfs.2019/20.9.3.502-504

\section{ARTICLE INFO}

Received 6. 4. 2019

Revised 13. 5. 2019

Accepted 30. 5. 2019

Published 1. 12. 2019

Short communication

open $\mathcal{O}$ access

\begin{abstract}
MarA (the multiple antibiotic resistance activator) is a regulatory protein that plays a significant role in multidrug resistance in bacteria and archaea where cellular mechanisms such as DNA and protein synthesis are inhibited during dormancy and subsequently cells evade a sudden antibiotic stress. MarAs (125-127 residues) of species selected from ten genera of the family Enterobacteriaceae were selected for analyses. MarA consists of seven $\alpha$-helices where about $81 \%$ of the residues in helices are conserved. The helices and folds in MarA of Salmonella enterica serovar typhimurium can be further divided into two structurally similar and interconnected subdomains, each containing a HTH DNA-binding motif. The recognition helices, H3 and H6 of the motifs are fully conserved, which are inserted into the adjacent major groove segments of DNA. The sequences show high similarity (83.4-100 \%) with MarA of E. coli K-12 with fully resolved three-dimensional structure at $2.3 \AA$.
\end{abstract}

Keywords: Enterobacteriaceae, Helix-turn-helix, Multiple antibiotic resistance, Regulatory protein, Salmonella sp.

\section{INTRODUCTION}

Multiple antibiotic resistance (mar) locus is present in several genera of the family Enterobacteriaceae (Cohen et al., 1993). The marRAB operon in Escherichia coli is auto-activated by MarA (Alekshun and Levy, 1997), an Ara$\mathrm{C}$ family prokaryotic dual regulator, which is known to transcriptionally activate or repress about sixty genes in response to multiple environmental stresses (Barbosa and Levy, 2000). This regulation occurs when MarA binds as a monomer to the promoter region at an asymmetric, degenerate 20-bp DNA sequence, the marbox (Martin and Rosner, 2002). Activation or repression depends upon the orientation and position of the marbox within the promoter (Schneiders et al., 2004).

Rhee et al. (1998) presented the first crystal structure of MarA of E. coli in complex with its associated DNA-binding site (PDB ID 1bl0). Overall structure of MarA-DNA complex consists of seven $\alpha$-helices organized into two structurally similar subdomains, each containing a helix-turn-helix (HTH) DNAbinding motif. HTH motif is ubiquitous and has been detected in many transcriptional regulators (Grishin, 2000). MarA binds to DNA segment by inserting helix-3 and helix-6 (the recognition helices) of the two subdomains into the two adjacent major groove segments of DNA. Helix-4 (the linker or central helix) controls the orientation and extent of this binding where the distance constraint results in bending the DNA by about 35 degrees (Rhee et al., 1998).

Salmonella enterica subsp. enterica serovar typhimurium is a rod-shaped, Gramnegative, flagellated facultative anaerobe, mostly present in the gastrointestinal tract (Feasey et al., 2012) causing diarrhea, abdominal cramps, fever and vomiting. This bacterium has a wide range of animal hosts including birds, cattle, many domesticated animals and humans. Since 2016, hundreds of cases of outbreak of extensively drug- resistant (XDR) typhoid have been reported in Pakistan (Chatham-Stephens et al., 2019). The mar locus, functionally similar to that of E. coli, has been identified in S. enterica (Randall and Woodward, 2001). In the present study, MarAs from selected species of ten genera of the family Enterobacteriaceae were selected for phylogenetic analysis and conserved helices and residues, with a special emphasis on MarA of S. enterica subsp. enterica serovar typhimurium. In addition, three-dimensional structure of MarA and MarA-DNA complex is also proposed.

\section{MATERIAL AND METHODS}

Protein sequences of MarAs of bacterial strains from ten selected genera (Citrobacter, Cronobacter, Enterobacter, Erwinia, Escherichia, Klebsiella,
Kluyvera, Proteus, Salmonella, and Shigella) of the family Enterobacteriaceae were obtained from NCBI (http://www.ncbi.nlm.nih.gov). NCBI's conserved domain database (CDD; Marchler-Bauer et al., 2017) was used to annotate functional units and conservations in MarAs. Structural elements were assigned with SCRATCH protein predictor (Cheng et al., 2005).

Pair-wise and multiple sequence alignments were made with CLUSTAL X 2.1 (Larkin et al., 2007). Sequence identity was determined using BioEdit 7.2.5 (Hall, 1999). The phylogenetic tree was constructed using the neighbor-joining method (Saito and Nei, 1987) based on 1000 replications. The evolutionary distances were computed using the Poisson-correction (PC) method (Zuckerkandl and Pauling, 1965) with MEGA7 (Kumar et al., 2016). Threedimensional structures of MarA and MarA-DNA complex were produced using the program VMD 1.9.3 (Humphrey et al., 1996).

\section{RESULTS AND DISCUSSION}

Figure 1 shows the marRAB operon in representative species from ten genera of the family Enterobacteriaceae. This operon consists of three genes. marA encodes transcriptional activator of genes involved in the multiple antibiotic resistance phenotype. marB encodes multiple antibiotic resistance regulatory periplasmic protein while marR encodes repressor protein involved in regulation of both antibiotic resistance and oxidative stress genes. An inner membrane protein MarC, which contains six predicted transmembrane domains, was originally thought to be involved in multiple antibiotic resistance. However, $\operatorname{mar} C$ promoter does not contain the MarR binding site. Similarly, $\operatorname{mar} C$ mutations do not lead to any change in susceptibility of strains to a number of antibiotics and oxidative stress agents (McDermott et al., 2008). 


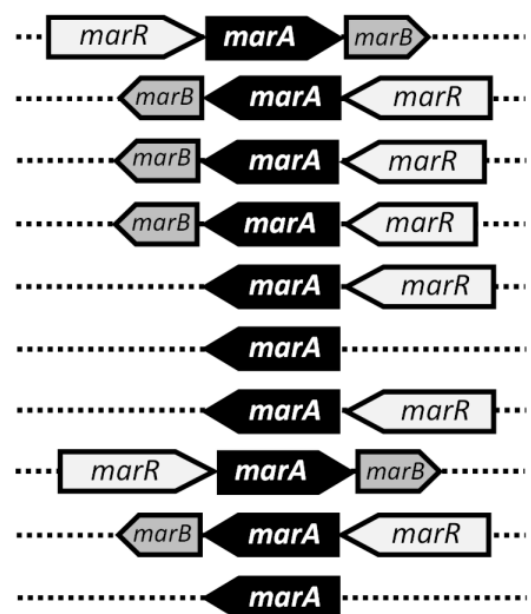

Escherichia col

Shigella flexneri

Salmonella enterica

Citrobacter freundii

Klebsiella pneumoniae

Proteus sp.

Enterobacter hormaechei

Kluyvera ascorbata

Cronobacter sakazakiia

Erwinia teleogrylii

Figure 1 The $\operatorname{mar} R A B$ operon in representative species from ten genera of the family Enterobacteriaceae. Only identified genes of the operon are shown as arrows. Description of the three genes is given in text.

Conserved sequences have slower rate of mutation and they maintain the structure or function of protein or a domain by natural selection. The selected MarAs contain 125 to 127 amino acid residues. Structural elements annotation by SCRATCH indicated that MarA of S. enterica is composed of seven $\alpha$-helices (H1 to $\mathrm{H} 7)$ while CDD inferred that MarA contains helix-turn-helix (HTH) DNA-binding motifs and belongs to AraC subfamily of transcriptional regulator proteins. A total of eleven hydrogen bonds between amino acid residues were identified in these helices. For ten genera of Enterobacteriaceae, sequence alignment of residues comprising these seven helices is shown in Figure 2. As indicated, $\mathrm{H} 3, \mathrm{H} 5$ and $\mathrm{H} 6$ are fully conserved in all the selected species of the ten genera. Especially, $\mathrm{H} 3$ and $\mathrm{H} 6$ are reported to be the recognition helices involved in DNA-binding in adjacent major grooves (Rhee et al., 1998). This conservation indicates importance of these residues to confer function of MarA as a DNAbinding protein. As MraA of S. enterica does not contain any cysteine residue, disulfide bonds are not formed.

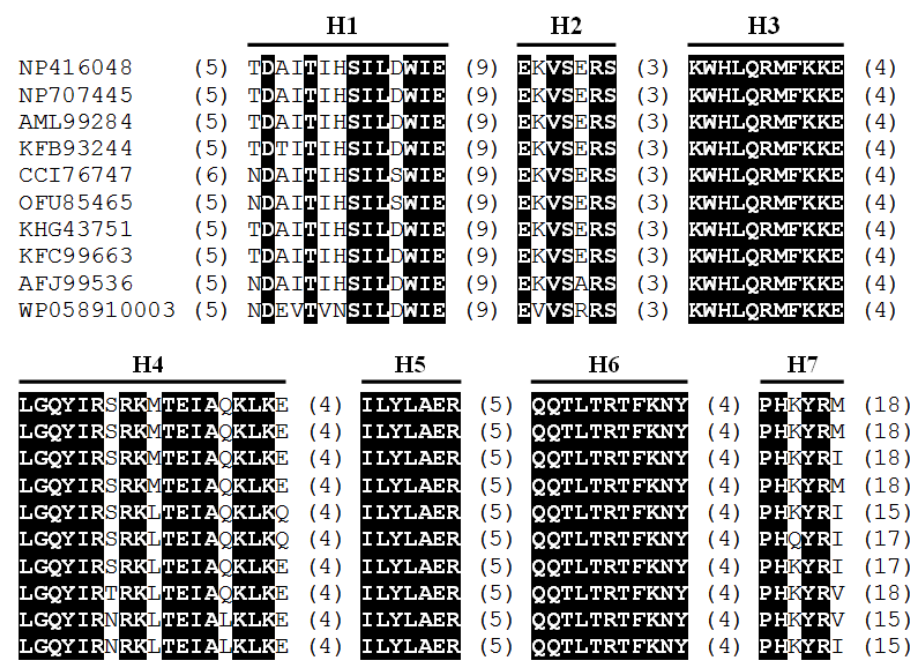

Figure 2 Sequence alignment of MarAs from selected species of genera of Enterobacteriaceae. Blocks $(\mathrm{H} 1-\mathrm{H} 7)$ correspond to seven $\alpha$-helices. Numbers within parentheses indicate number of residues between protein termini and proximal and distal aligned blocks while numbers between individual blocks indicate number of residues separating them. Identical amino acid residues are highlighted in black. Accession numbers correspond to the species as mentioned in Figure 3.

The phylogenetic relationship of MarA from representative species of the ten genera is shown in Figure 3. As indicated, ther is $94.4 \%$ sequence identity between MarAs of E. coli str. K-12 and S. enterica subsp. enterica serovar typhimurium. MarA of Pantoea ananatis LMG20103 was used as outgroup with only $43.3 \%$ and $42.6 \%$ sequence identity with the MarAs of $E$. coli and $S$. enterica, respectively.

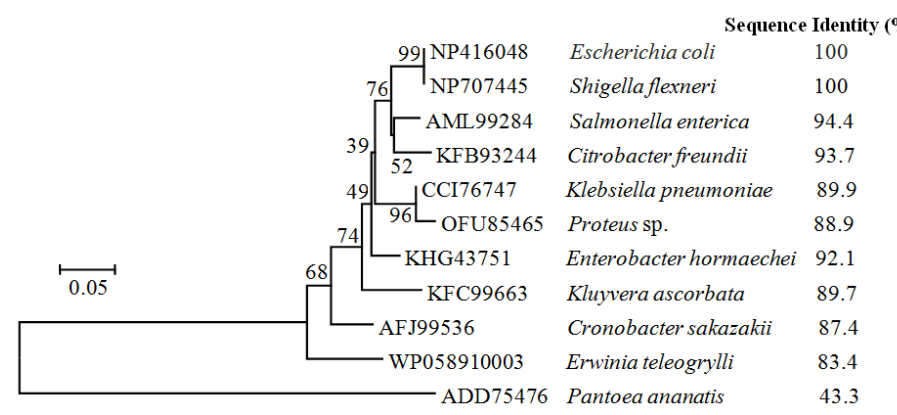

Figure 3 Consensus phylogenetic tree of MarAs of selected species that belong to ten genera of the family Enterobacteriaceae. Accession numbers are indicated for each species. MarA of Pantoea ananatis (ADD75476) serves as outgroup The tree is drawn to scale where the scale bar indicates percent divergence (distance) in the units of the number of amino acid substitutions per site Evolutionary analyses were conducted in MEGA7.

Three-dimensional structure of MarA of S. enterica is represented as Figure 4A where seven $\alpha$-helices are shown as cylinders H1to H7. The helices and folds in MarA can be further divided into two structurally similar interconnected subdomains, separated by 27 residues. This $\mathrm{C}$ - and N-subdomain (residues 6-49 and 77-109, respectively) is connected by a relatively bigger helix, H4 (19 residues) called the linker helix. Each of these subdomains contains a HTH DNA-binding motif (residues 35-38 and 84-88). These turns between $\mathrm{H} 2$ and $\mathrm{H} 3$, and between $\mathrm{H} 5$ and $\mathrm{H} 6$ are shown as T1 and T2, respectively in Figure 4 (A B). Sequence of T2 (residues 84-88) was also conserved in all the selected species. The orientation of the recognition helices, $\mathrm{H} 3$ and H6, is imposed by the linker helix (H4) where the protein binds to one face of the DNA. As indicated by Figure 4B, H3 and $\mathrm{H} 6$ of the motifs are inserted into the adjacent major groove segments.

A

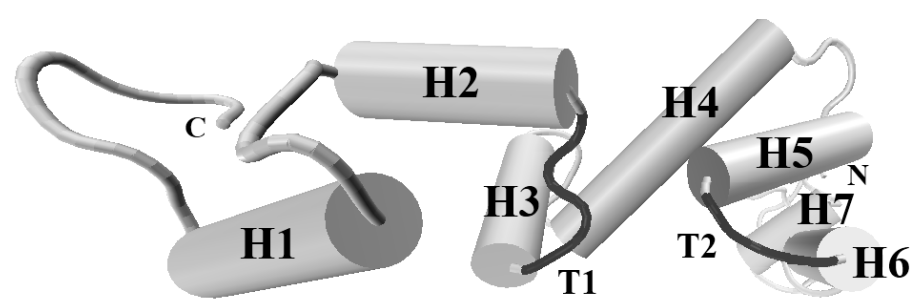

$\mathbf{B}$

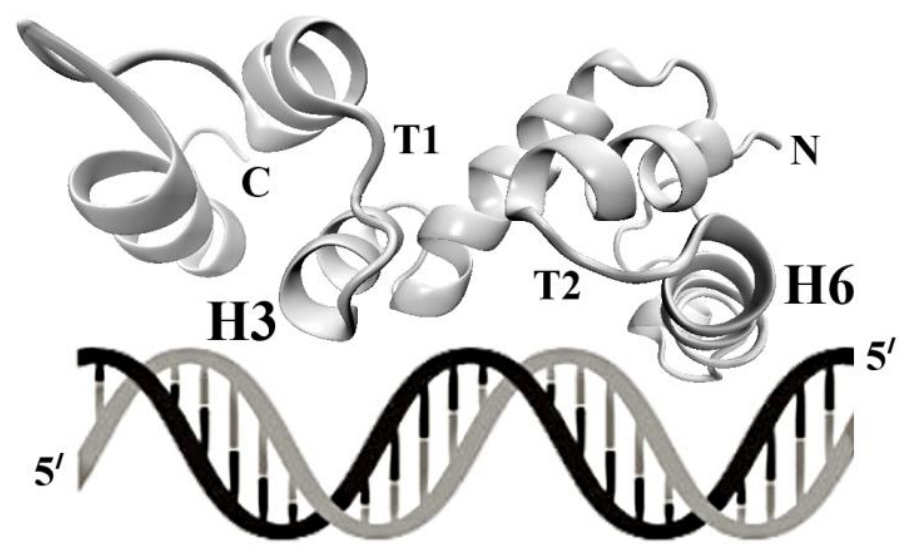

Figure 4 (A) The overall structure of MarA monomer of $S$. enterica. $\mathrm{H}$ corresponds to helix while $\mathrm{T} 1$ and $\mathrm{T} 2$ (shown in black) correspond to turns in Cand N-subdomain, respectively. (B) A ribbon diagram of MarA and its complex with DNA representing insertion of the recognition helices into adjacent major grooves.

In conclusion, MarAs of Enterobacteriaceae share the seven $\alpha$-helix structure with two subdomains containing HTH DNA-binding motifs. More than $80 \%$ of the residues of helices are conserved. The recognition helices are fully conserved that are reported to be inserted into the two adjacent major grooves of DNA. 


\section{REFERENCES}

Alekshun, M.N., \& Levy, S.B. (1997). Regulation of chromosomally mediated multiple antibiotic resistance: the mar regulon. Antimicrobial Agents and Chemotherapy, 41, 2067-2075.

Barbosa, T.M., \& Levy, S.B. (2000). Differential expression of over 60 chromosomal genes in Escherichia coli by constitutive expression of MarA Journal of Bacteriology, 182, 3467-3474. https://dx.doi.org/10.1128/JB.182.12.3467-3474.2000

Chatham-Stephens, K., Medalla, F., Hughes, M., Appiah, G.D., Aubert, R.D., Caidi, H., ... \& Friedman, C.R. (2019). Emergence of extensively drugresistant Salmonella typhi infections among travelers to or from Pakistan - United States, 2016-2018. Morbidity and Mortality Weekly Report, 68, 11-13. https://dx.doi.org/10.15585/mmwr.mm6801a3

Cheng, J., Randall, A.Z., Sweredoski, M.J., \& Baldi, P. (2005). SCRATCH: a protein structure and structural feature prediction server. Nucleic Acids Research, 33, W72-W76. https://dx.doi.org/10.1093/nar/gki396

Cohen, S.P., Yan, W., \& Levy, S.B. (1993). Bacterial genetics, antibiotic usage, and public policy: the crucial interplay in emerging resistance. Journal of Infectious Diseases, 168, 484-488. https://dx.doi.org/10.1093/infdis/168.2.484

Feasey, N.A., Dougan, G., Kingsley, R.A., Heyderman, \& R.S., Gordon, M.A (2012). Invasive non-typhoidal salmonella disease: an emerging and neglected tropical disease in Africa. Lancet, 379, 2489-2499. https://dx.doi.org/10.1016/S0140-6736(11)61752-2

Grishin, N.V. (2000). Progress in nuclear acid research and molecular biology. $\begin{array}{llll}\text { Nucleic Acids } & \text { Research, 28, 229-2233. }\end{array}$ https://dx.doi.org/10.1093/nar/28.11.2229

Hall, T.A. (1999). BioEdit: a user-friendly biological sequence alignment editor and analysis program for Windows 95/98/NT. Nucleic Acids Symposium Series, 41, 95-98.

Kumar, S., Stecher, G., \& Tamura, K. (2016). MEGA7: molecular evolutionary genetics analysis version 7.0 for bigger datasets. Molecular Biology and Evolution, 33, 1870-1874. https://dx.doi.org/10.1093/molbev/msw054

Larkin, M.A., Blackshields, G., Brown, N.P., Chenna, R., McGettigan, P.A., McWilliam, H., ... \& Higgins, D.G. (2007). Clustal W and Clustal X version 2.0 Bioinformatics, 23, 2947-2948. https://dx.doi.org/10.1093/bioinformatics/btm404 Marchler-Bauer, A., Derbyshire, M.K., Gonzales, N.R., Lu, S., Chitsaz, F., Geer, L.Y., ... \& Bryant, S.H. (2017). CDD: NCBI's conserved domain database. Nucleic Acids Research, 43, 222-226. https://dx. doi.org/10.1093/nar/gku1221

Martin, R.G., Jair, K.-W., Wolf, R.E., \& Rosner, J.L. (1996). Autoactivation of the $\operatorname{marR} A B$ multiple antibiotic resistance operon by the MarA transcriptional activator in Escherichia coli. Journal of Bacteriology, 178, 2216-2223. https://dx.doi.org/10.1128/jb.178.8.2216-2223.1996

Martin, R.G., \& Rosner, J.L. (2002).Complex formation between activator and RNA polymerase as the basis of transcriptional activation by MarA and SoxS in Escherichia coli. Molecular Microbiology, 44, 1611-1624. https://dx.doi.org/10.1046/j.1365-2958.2002.02985.x

McDermott, P.F., McMurry, L.M., Podglajen, I., Dzink-Fox, J.L., Schneiders, T., Draper, M.P., \& Levy, S.B. (2008). The marC gene of Escherichia coli is not involved in multiple antibiotic resistance. Antimicrobial Agents and Chemotherapy, 52, 382-383. https://dx.doi.org/10.1128/AAC.00930-07

Randall, L.P., \& Woodward, M.J. (2001). Multiple antibiotic resistance (mar) locus in Salmonella enteric serovar Typhimurium DT104. Applied and Environmental Microbiology, $\quad 67, \quad 1190-1197$ https://dx.doi.org/10.1128/AEM.67.3.1190-1197.2001

Rhee, S., Martin, R.G., Rosner, J.L., \& Davies, D.R. (1998). A novel DNA binding motif in MarA: the first structure of an AraC family transcriptional activator. Proceedings of the National Academy of Sciences of the United States of America, 95, 10413-10418.

Saito, N., \& Nei, M. (1987). The neighbor-joining method: a new method for the reconstructing phylogenetic trees. Molecular Biology and Evolution, 4, 406-425 https://dx.doi.org/10.1093/oxfordjournals.molbev.a040454

Schneiders, T., Barbosa, T.M., McMurry, L.M., \& Levy, S.B. (2004). The Escherichia coli transcriptional regulator MarA directly represses transcription of purA and hdeA. Journal of Biological Chemistry, 279, 9037-9042. https://dx.doi.org/10.1074/jbc.M313602200

Zuckerkandl, I., \& Pauling, L. (1965). Evolutionary divergence and convergence in proteins. In V. Bryson \& H.J. Vogel (Ed.), Evolving genes and proteins. Academic Press, New York. pp. 97-166. ISBN 9781483266305 http://dx.doi.org/10.1016/B978-1-4832-2734-4.50017-6 